\title{
Erratum to: Urinary Protein Markers for the Detection and Prognostication of Urothelial Carcinoma
}

\author{
Tibor Szarvas, Péter Nyirády, Osamu Ogawa, Hideki Furuya, \\ Charles J. Rosser, and Takashi Kobayashi
}

Erratum to:

Chapter 19 in: Wolfgang A. Schulz et al. (eds.), Urothelial Carcinoma: Methods and Protocols, Methods in Molecular Biology, vol. 1655, https://doi.org/10.1007/978-1-4939-7234-0_19

The original version of this chapter was inadvertently published with incorrect order of authors. The correct order is updated in the current version as Tibor Szarvas, Péter Nyirády, Osamu Ogawa, Hideki Furuya, Charles J. Rosser, and Takashi Kobayashi. 